\title{
Errata
}

\section{An Iterative Process to Calculate the SCF Open Shell Orbitals}

Renato Colle, Raffaele Montagnani, Pierluigi Riani and Oriano Salvetti

Theoret. Chim. Acta (Berl.) 48, 251-256 (1978).

In the expression of the $\widetilde{F_{d}}$ operator (page 254 , third line from the bottom), the multiplication by the occupation number has been omitted. Therefore this formula must be read:

$$
\mathscr{F}_{d}=2\left(h+\sum_{j=1}^{m}\left(2 \mathscr{F}_{j}-\mathscr{K}_{j}\right)+\mathscr{J}_{a}+\mathscr{H}_{b} \pm \frac{1}{2}\left(\mathscr{K}_{a}+\mathscr{K}_{b}\right)\right)
$$

Received July 17, 1978 